\title{
Investigation of the incidence of Eustachian tube dysfunction in patients with sinonasal disease*
}

\author{
C.E. Rennie', M. Gutierrez², Y. Darby', V.J. Lund \\ 'Royal National Throat Nose and Ear Hospital, ENT, London, United Kingdom \\ ${ }^{2}$ University of North Carolina, Medical student, North Carolina, USA
}

Rhinology Online, Vol 1: 85 - 89, 2018

http://doi.org/10.4193/RHINOL/18.050

*Received for publication:

August 12, 2018

Accepted: August 14, 2018

Published: August 19, 2018

\begin{abstract}
Background: Rhinosinusitis is characterised by inflammation affecting the respiratory mucosa of the nose and sinuses. Since the Eustachian tube and the middle ear cavity are also lined by the same mucosa, it is likely that the pathophysiological processes that give rise to rhinosinusitis will also affect these areas. Eustachian tube dysfunction (ETD) is a common condition, but it is often dismissed as a "minor" symptom in rhinology patients.
\end{abstract}

Objective: The aim of this study was to determine the frequency of otologic symptoms in patients attending the rhinology clinic. The seven-item Eustachian Tube Dysfunction Questionnaire (ETDQ-7), a validated disease-specific instrument was used to assess symptoms with respect to ETD7.

Study design: 119 patients attending the rhinology outpatient clinic completed ETDQ-7, a SNOT 22 and had their PNIF measured.

Results: The results showed a significantly higher rate of ETD $(p<0.01$ paired t-test) in the rhinology patients (mean score 3.1, SD 1.64) as compared to a control population (mean 1.3, SD 0.3).

Conclusion: Eustachian tube dysfunction is more common in rhinology patients then the general population, and within the rhinology population. The classification of this as a "minor" symptom of rhinosinusitis should be re-evaluated and the effect of treatment of sinonasal disease on ETD needs to be investigated.

Key words: Eustachian tube dysfunction, paranasal sinuses, rhinosinusitis, questionnaire

\section{Introduction}

Rhinosinusitis is characterised by inflammation affecting the respiratory mucosa of the nose and sinuses. The Eustachian tube and the middle ear cavity are also lined by the same mucosa. It is therefore, likely that the pathophysiological processes that give rise to rhinosinusitis will also affect these areas.

Although the connection between these adjacent areas has been realised for decades, there is a lack of research examining this interaction and the effects of any interventions. Most studies in this area focus on otitis media with effusion in children (1-3).
Eustachian tube dysfunction (ETD) is a common symptom, it is estimated to have a prevalence of $0.9 \%$ in the UK population ${ }^{4)}$, however it is often dismissed as a "minor" symptom in rhinology patients. This may in part be due to the difficulty diagnosing ETD as its symptoms are often vague and non-specific, also there is a perceived lack of efficacy of any treatment for ETD. Until recently there has been no consensus on the definition and diagnosis of this disorder ${ }^{(5,6)}$. The lack of definition of ETD along with the paucity of outcome measures has hampered our ability to determine the epidemiology and efficacy of treatments for ETD. 
The development of the first international consensus on the definition, clinical presentation and diagnosis of ETD should enable better studies of the epidemiology of Eustachian tube dysfunction, psychosocial impact, and treatment ${ }^{\left({ }^{6}\right)}$.

The Eustachian tube has three main functions:

1. Pressure equalisation and ventilation of the middle ear

2. Mucociliary clearance of secretions from the middle ear

3. Protection of the middle ear from sounds as well as from pathogens and secretions from the nasopharynx ${ }^{(7,8)}$.

In health the Eustachian tube is normally closed but is pulled open briefly by the paratubal muscles during swallowing or yawning, which enables the above functions to take place ${ }^{(9,10)}$. Disruption of this intermittent opening leads to ETD with the typical symptoms of ear fullness, 'popping' or 'crackling', discomfort or pain, muffled hearing or tinnitus ${ }^{5,111)}$.

Over the last 40 years there have been a number of attempts to develop an objective measure for ETD ${ }^{(12)}$ however, as yet no single test has been found to be a reliable diagnostic tool ${ }^{(13,14)}$. The recently developed Eustachian Tube Dysfunction Questionnaire (ETDQ- 7) scores symptoms of Eustachian tube dysfunction and is currently the only patient-reported outcomes tool to have undergone initial validation studies ${ }^{(6)}$.

Therefore, in this study we use the ETDQ-7 to determine the incidence of Eustachian tube dysfunction symptoms in patients attending a tertiary referral rhinology clinic. The ETDQ-7 is short and simple to use, and the respondent burden is minimal. It has been shown to be reliable and valid for the cross-sectional assessment of ETD-related symptoms in adults. We have compared the results in our rhinology population to literature quoted values for ETD patient and control groups from the validation studies.

\section{Methods}

Patients were prospectively and consecutively enrolled from the clinical practice of the senior author (VJL) over a 6 month period in the latter half of the year to minimise effects of seasonal allergy (Appendix). All subjects were outpatients who were referred with chronic rhinologic symptoms (>3months) to a tertiary referral centre and participated as part of an institutional audit. All patients included in this study were at least 18 years old. All had symptoms and signs consistent with a diagnosis of chronic rhinosinusitis ${ }^{(15)}$ but patients with known otological disease and previous ear surgery were excluded. Each patient completed an ETDQ-7, a SNOT 22, a visual analogue score (VAS) for nasal obstruction, and had their peak nasal inspiratory flow (PNIF) measured as part of their initial assessment. The ETDQ-7 is comprised of seven questions on ETD symptoms each of which is
Table 1. Eustachian tube dysfunction questionnaire ETDQ-7.

$\begin{aligned} & \text { Over the past } 1 \text { month, how much has each of } \\
& \text { the following been a problem for you? }\end{aligned}$
\begin{tabular}{llllllll}
\hline No Problem & Moderate Problem & $\begin{array}{c}\text { Severe } \\
\text { Problem }\end{array}$ \\
\hline 1. Pressure in the ears? & 1 & 2 & 3 & 4 & 5 & 6 & 7 \\
2. Pain in the ears? & 1 & 2 & 3 & 4 & 5 & 6 & 7 \\
3. A feeling that your ears are clogged or "under water"? & 1 & 2 & 3 & 4 & 5 & 6 & 7 \\
4. Ear symptoms when you have a cold or sinusitis? & 1 & 2 & 3 & 4 & 5 & 6 & 7 \\
5. Crackling or popping sounds in the cars? & 1 & 2 & 3 & 4 & 5 & 6 & 7 \\
6. Ringing in the ears? & 1 & 2 & 3 & 4 & 5 & 6 & 7 \\
7. A feeling that your hearing is muflled? & 1 & 2 & 3 & 4 & 5 & 6 & 7
\end{tabular}

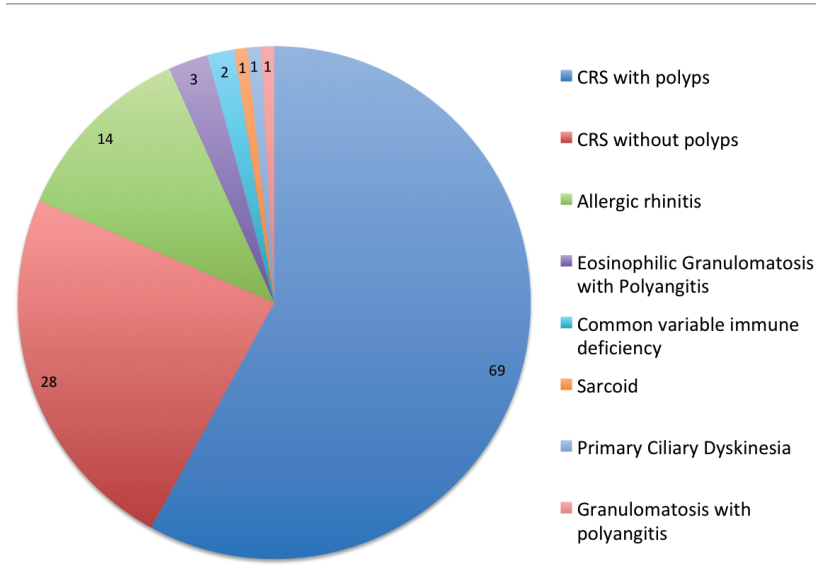

Figure 1. Distribution of pathology within the rhinology patient group.

scored out of 7 for severity over the last month (Table 1). This differs from the SNOT 22 questionnaire, which scores 22 symptoms of rhinosinusitis out of 5 over the previous 2 weeks.

\section{Results}

A total of 119 patients were enrolled for the study, 65 female and 54 male, with a mean age of 52 . Over half (54\%) of the patients had had previous endoscopic sinus surgery, which is typical of the tertiary referral practice. There was also a wide range of pathologies within the patient group also reflecting the nature of a tertiary referral practice (Figure 1).

The ETDQ-7 results for the rhinology patients were compared to the results quoted in the literature for control populations and patients with ETD (Table 2, Figures 2 and 3) (11). The overall ETDQ7 score among the 119 rhinology patients was significantly greater than the score among the literature control group $(\mathrm{P}<$ 0.001). The mean (standard deviation) overall score was 2.8 (1.6) for the rhinology patients, 4.0 (1.1) for the ETD group and 1.3 (0.3) for the control group. The mean individual score for each of the seven items of the ETDQ-7 was significantly greater for the rhinology group compared to the control group. As expected the rhinology patients scored highest on Question 4 of the ETDQ-7 "ear symptoms when you have a cold or sinusitis?". 
Table 2. Eustachian Tube Dysfunction Scores by Item. SD = standard deviation. * Literature values given by McCoul et al. (11).

\begin{tabular}{lccc|}
\multicolumn{1}{|c}{ Item } & $\begin{array}{c}\text { Rhinology } \\
\text { patients } \\
\text { Mean (SD) }\end{array}$ & $\begin{array}{c}\text { Control } \\
\text { Group* } \\
\text { Mean (SD) }\end{array}$ & $\begin{array}{c}\text { ETD } \\
\text { Group * } \\
\text { Mean (SD) }\end{array}$ \\
\hline 1. Pressure & $2.6(1.8)$ & $1.1(0.4)$ & $4.3(1.5)$ \\
\hline 2. Pain & $2.3(1.7)$ & $1.3(0.6)$ & $3.2(1.7)$ \\
\hline 3. Feeling clogged & $2.8(1.9)$ & $1.2(0.5)$ & $4.8(1.5)$ \\
\hline $\begin{array}{l}\text { 4. Cold/sinusitis } \\
\text { problems }\end{array}$ & $3.4(2.8)$ & $2.2(1.1)$ & $5.0(1.6$ \\
\hline $\begin{array}{l}\text { 5. Crackling or } \\
\text { popping }\end{array}$ & $2.8(2.0)$ & $1.1(0.4)$ & $4.0(1.8)$ \\
6. Ringing & $2.6(2.1)$ & $1.1(0.3)$ & $3.2(2.1)$ \\
\hline 7. Feeling muffled & $3.1(2.1)$ & $1.3(0.3)$ & $4.8(1.5)$ \\
\hline
\end{tabular}

In validity studies the optimal total item score cutpoint of $\geq 14.5$ versus $<14.5$ (for categorising a patient as having ETD) provided $100 \%$ sensitivity and $100 \%$ specificity. This equated to an ETDQ7 mean item score of $\geq 2.1$ to indicate the presence of ETD. 74 of the 119 rhinology patients in this study had total item scores $\geq 14.5$, meaning that $62 \%$ of these rhinology patients would be diagnosed as having ETD based on this outcome measure.

SNOT 22 scores of $>28.5$ were associated with higher ETDQ-7 scores, which is not surprising as the SNOT 22 has 3 otological questions pertaining to ear fullness, dizziness and ear pain/ pressure which overlaps with the ETDQ-7 questions 1, 2 and 3. (Table 2) There is also an overlap between Question 4 of the ETDQ-7 ("ear symptoms when you have a cold or sinusitis") with symptoms of rhinosinusitis. The SNOT-22 alone had a moderate specificity and sensitivity for detecting ETD on validity testing11, although the ETDQ-7 performed significantly better as a disease-specific instrument.

VAS scores $>5$ for nasal obstruction were associated with higher ETDQ-7 scores and NIPF scores $<80$ were also associated with higher ETDQ-7 scores.

\section{Discussion}

These results illustrate that ETD, as manifested by otologic symptoms, is common in patients attending the rhinology clinic. This has been confirmed recently in a combined multi-centre cohort of 131 patients with CRS based on responses to the SNOT-22 questions on ear fullness and ear pain ${ }^{(16)}$. Therefore, the classification of ETD as a "minor" symptom of rhinosinusitis should be re-evaluated.

What remains to be determined is if treatment for sinus symptoms will improve the symptoms of Eustachian tube dysfunction? An earlier retrospective questionnaire study by Stoikes et al. suggests the ETD symptoms associated with CRS improve or resolve in the majority of patients undergoing endoscopic si-

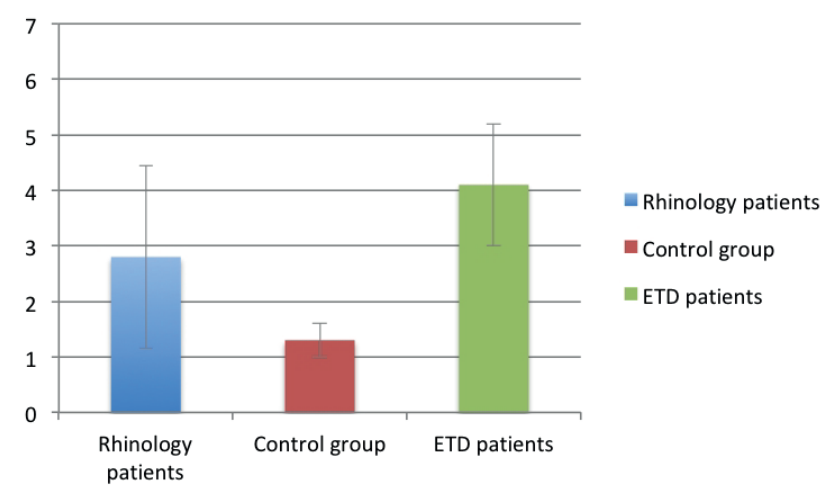

Figure 2. Mean ETDQ-7 scores. There was a significantly higher rate of ETD symptoms ( $p<0.01$ paired $t$-test) in the rhinology patients (mean score 3.1, SD 1.64) as compared to a control population (mean 1.3, SD 0.3). The error bars show the standard deviation.

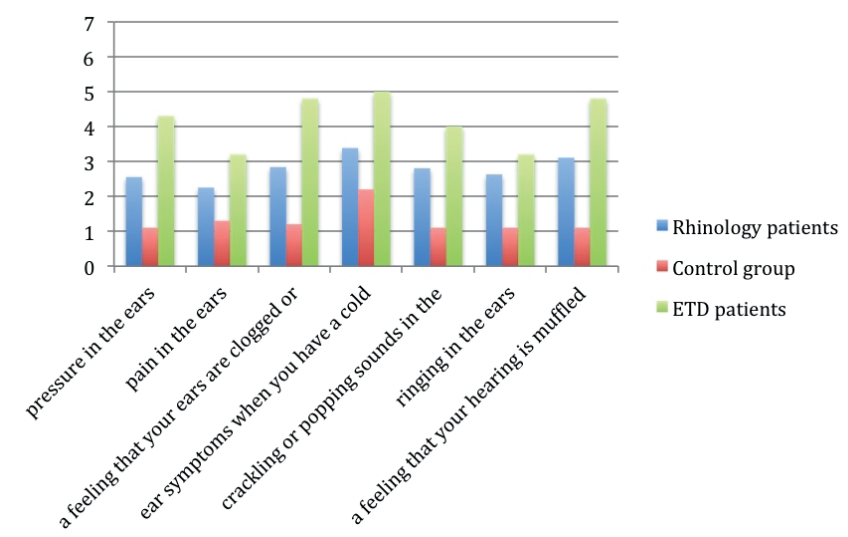

Figure 3. Mean ETDQ-7 scores by item.

nus surgery ${ }^{(17)}$. Maniakas et al. also confirmed that for most CRS patients, ETD symptoms will decrease post-endoscopic sinus surgery to a level comparable with a non-CRS population ${ }^{(16)}$. The use of the ETDQ-7 has the potential to enhance clinical care by highlighting the impact of ETD as well as guiding and evaluating appropriate management. Further prospective testing of patients being treated for ETD may establish the ETDQ-7 in the assessment of treatment outcomes.

\section{Limitations of this study}

A potential confounding factor is that a small number of the rhinology patients in this cohort had systemic disease with known otological manifestations, which could bias these results. As expected higher ETDQ-7 scores were seen in patients with primary ciliary dyskinesia (PCD), eosinophilic granulomatosis with polyangitis (EGPA) and immunodeficiency (Figure 4) who nonetheless were suffering from chronic rhinosinusitis. Therefore, further analysis was undertaken excluding these patients and the results still demonstrated significantly higher scores in the rhinology population as compared to control groups. 


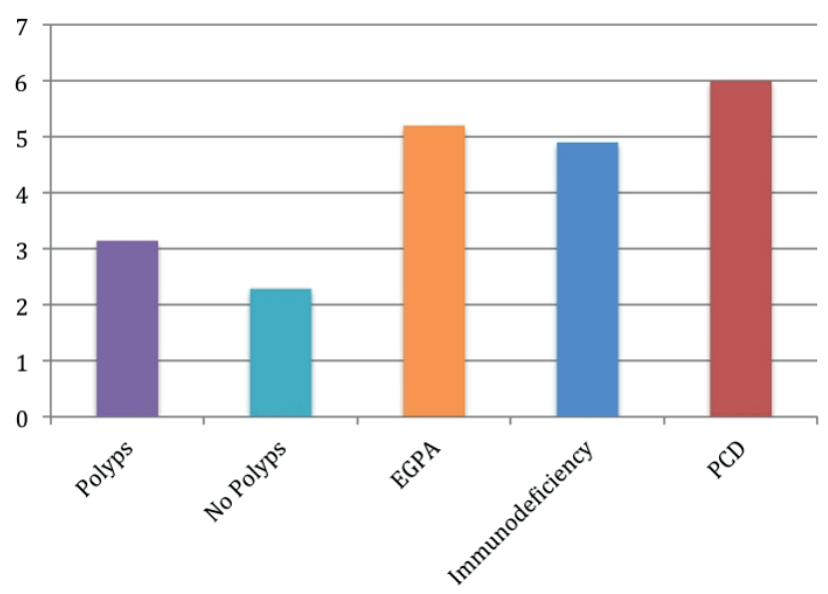

Figure 4. Mean ETDQ-7 score by diagnosis. The number of patients with each diagnosis is as follows; Polyps (CRSwNP) 69, No polyps (CRSsNP) 28, EGPA (Eosinophilic granulomatosis with polyangitis) 3, Immunodeficiency 2 and PCD (Primary ciliary dyskinesia) 1.

The recent consensus document on ETD recommends that the diagnosis of ETD be based on the presentation with symptoms of pressure disequilibrium in either ear, specifically symptoms of 'aural fullness' or 'popping' or discomfort/pain ${ }^{(6)}$. Evidence of negative pressure in the middle ear could be supported on clinical assessment, either by:

1. otoscopic evidence of tympanic membrane retraction and/or 2. tympanogram indicating negative middle ear pressure.

Unfortunately neither in this study nor that of Maniakas et al. ${ }^{(17)}$ the rhinology patients have not been assessed for evidence of negative pressure in the middle ear. However, the ETDQ-7 is a valid and reliable disease-specific symptom score for adult patients with ETD. The criterion validity for the test was established by the exclusive presence of normal tympanograms in control subjects and abnormal tympanograms in subjects with ETD. The advantage of the ETDQ-7 is that it is quick and easy to administer in the clinical setting and is simple for the patients to fill out. Another limitation of the study related to the ETDQ-7 is that the questions are based on disease burden and timing of events. In addition, whether they may be intermittent is not represented and therefore, the questionnaire may underestimate the incidence of ETD. Furthermore, there is a possibility of bias by comparing our patients with historical controls from the United States rather than providing our own control data.

\section{Conclusion}

Symptoms of eustachian tube dysfunction are prevalent in the rhinology patient population and are often overlooked in sinonasal-orientated outpatient consultations. The development of a consensus on the definition and diagnosis of ETD and its adoption by the ENT community should lead to an improvement in the diagnosis of ETD and will provide the basis for further research into the epidemiology and treatment of ETD. The development and validation of disease specific patient recorded outcome measures such as the ETDQ-7 allows comparison of the efficacy of treatments for ETD and an understanding of the interaction between the nose and Eustachian apparatus could lead to a better overall management of our patients.

\section{Key Points}

- Symptoms of eustachian tube dysfunction are more common in rhinology patients then in the general population.

- The ETDQ-7 is a simple easy to use validated disease specific outcome measure for ETD.

- The classification of this as a "minor" symptom of rhinosinusitis should be re-evaluated.

- The effect of treatment of sinonasal disease on ETD needs to be investigated.

\section{Authorship contribution}

MG, YD and CR were involved in the design and data collection for the study, VJL designed the study and edited the manuscript and approved the final version. CR processed the results, drafted the manuscript, reviewed the available literature and approved the final version.

\section{Availability of data and materials}

Curated by first author and available on reasonable request.

\section{Conflict of interest}

The authors declare that they have no competing interests.

\section{References}

1. Leo G, Piacentini E, Incorvaia C, Consonni D. Sinusitis and Eustachian tube dysfunction in children. Pediatr Allergy Immunol 2007; Supplement 18: 35-39.

2. Hong CK, et al. Effect of paranasal sinusitis on the development of otitis media with effusion: influence of eustachian tube function and adenoid immunity. Int J Pediatr Otorhinolaryngol 2008;72:1609-1618.
3. Fujita A, Honjo I, Kurata K, Gan I, Takahashi $H$. Refractory otitis media with effusion from viewpoints of eustachian tube dysfunction and nasal sinusitis. Am J Otolaryngol 1993;14: 187-190.

4. Browning GG, Gatehouse S. The prevalence of middle ear disease in the adult British population. Clin Otolaryngol Allied Sci 1992;17: 317-321.

5. Llewellyn A, et al. Interventions for adult
Eustachian tube dysfunction: a systematic review. Health Technol Assess 2014;18: 1-180, v-vi.

6. Schilder AG, et al. Eustachian tube dysfunction: consensus statement on definition, types, clinical presentation and diagnosis. Clin Otolaryngol 2015;40:407-411.

7. Bluestone CD. Eustachian Tube. Structure, Function, Role in Otitis Media, (BC Decker, Hamilton, London., 2005). 
8. Sade J, Ar A. Middle ear and auditory tube: middle ear clearance, gas exchange, and pressure regulation. Otolaryngol Head Neck Surg 1997;116:499-524.

9. Alper CM, Swarts JD, Singla A, Banks J, Doyle WJ. Relationship between the electromyographic activity of the paratubal muscles and eustachian tube opening assessed by sonotubometry and videoendoscopy. Arch Otolaryngol Head Neck Surg 2012;138: 741746.

10. Poe DS, Pyykko I, Valtonen H, Silvola J. Analysis of eustachian tube function by video endoscopy. Am J Otol 2000;21: 602607.

11. McCoul ED, Anand VK, Christos PJ. Validating the clinical assessment of eustachian tube dysfunction: The Eustachian Tube Dysfunction Questionnaire (ETDQ-7). Laryngoscope 2012;122: 1137-1141.
12. Holmquist J, Olen L. Evaluation of Eustachian tube function. J Laryngol Otol 1980;94: 15-23.

13. Norman G, et al. Systematic review of the limited evidence base for treatments of Eustachian tube dysfunction: a health technology assessment. Clin Otolaryngol 2014;39: 6-21.

14. Todd NW. There are no accurate tests for eustachian tube function. Arch Otolaryngol Head Neck Surg 2000;126: 1041-1042.

15. Fokkens W, Lund V, Mullol J, et al. European Position Paper On Rhinosinusitis And Nasal Polyps 2012 Rhinology Supplement 23:1298.

16. Maniakas A, Desrosiers M, Asmar M-H,et al. Eustachian tube symptoms are frequent in chronic rhinosinusitis and respond well to endoscopic sinus surgery. Rhinology 2018;56:118-121.
17. Stoikes NF, Dutton JM. The effect of endoscopic sinus surgery on symptoms of eustachian tube dysfunction. Am J Rhinol 2005;19;199-202.

Valerie J Lund

RNTNEH

Grays Inn Road

London WC1X 8DA

United Kingdom

Tel: +44 (0)7860 873726

E-mail:v.lund@ucl.ac.uk

\section{APPENDIX}

Inclusion and exclusion criteria for control group and ETD group McCoul et al (11).

"Patients were diagnosed as having ETD if they had a retracted or poorly mobile tympanic membrane on pneumatic otoscopy, with a history of at least two of the following symptoms in one or both ears over the previous 1 month period: aural fullness or pressure, a sensation of clogged or muffled hearing, recurrent or persistent middle ear effusion (defined as an effusion present on examinations at least 1 month apart), or the inability to rapidly self-equilibrate middle ear pressure following changes in ambient atmospheric pressure. Abnormal impedance audiometry was used as a criterion standard to verify the diagnosis at the time of enrolment. Exclusion criteria included surgery of the head or neck within 3 months; a history of radiation therapy to the head and neck; sino- nasal malignancy; evidence of acute upper respiratory infection, including sinusitis and acute otitis media; adenoid hypertrophy; nasal polyposis; cleft palate or history of cleft palate repair; craniofacial syndrome, including Down syndrome; cystic fibrosis; ciliary dysmotility syndrome; or other systemic immunodeficiency. A second group of patients who did not meet these inclusion criteria and who had presented with medical complaints not related to ETD were consecutively enrolled for use as a control group. Presenting complaints for these patients included voice disturbance, tonsil hypertrophy, and intraoral lesions. All of these patients had a normal examination of the tympanic membrane, middle ear, nasal cavity, and nasopharynx. Normal impedance audiometry was used as a criterion standard to verify the absence of ETD." 